\title{
Juego serio para entrenar habilidades auditivas en niños con discapacidad auditiva
}

\section{Serious Game to Train Auditory Skills for Children with Hearing Impairments}

\author{
Cristina Manresa-Yee1. (iD), Ramon Mas-Sansó ${ }^{1}$, Sandra Cano $^{2}$ \\ 1 Departamento de Ciencias Matemáticas e Informática, Universitat de les Illes Balears, Palma, España. \\ 2 Universidad de San Buenaventura de Cali, Cali, Colombia. \\ cristina.manresa@uib.es, ramon.mas@uib.es, sandra.cano@gmail.com
}

(Recibido: 7 Diciembre 2017; aceptado: 17 Enero 2018)

\begin{abstract}
Resumen. En este trabajo se presenta el diseño de un juego serio, cuyo objetivo es ejercitar la identificación y la discriminación auditiva en niños con discapacidades auditivas, tanto en aquellos que utilizan implantes cocleares como audífonos. En el diseño se han tenido en cuenta aspectos de diseño emocional, metodologías de desarrollo de juegos serios y las actividades que llevan a cabo los educadores con los niños para trabajar estas habilidades auditivas.
\end{abstract}

Palabras clave: Niños, Discapacidad auditiva, Discriminación e identificación auditiva, Educación, Diseño, Juego Serio.

\begin{abstract}
This paper presents the design of a serious game whose objective is to work the auditory identification and discrimination in children with hearing impairments, both for children using cochlear implants or auditory aids. The design takes into account emotional design issues, serious game development methodologies, and the activities carried out by educators and therapists with children to work on these auditory skills.
\end{abstract}

Keywords: Children, Hearing impairment, Auditory Discrimination and Identification, Education, Design, Serious game.

\section{Introducción}

Según la OMS (Organización Mundial de la Salud), 360 millones de personas en todo el mundo padecen pérdida de audición discapacitante, de las cuales 32 millones son niños entre 0 y 14 años. En los niños, se entiende que la audición es una discapacidad cuando la pérdida de audición es superior a $30 \mathrm{~dB}$ en el oído con mejor audición [1].

La audición discapacitante tiene una serie de consecuencias a nivel funcional, como déficits y retrasos en el desarrollo [2], una limitada capacidad para comunicarse o el retraso en el desarrollo del habla. Además, los niños con problemas auditivos desatendidos también pueden sufrir mayor fracaso escolar y, por tanto, necesitar mayor asistencia educativa. También existen consecuencias a nivel social y emocional en los niños con problemas auditivos, debido a factores como el aislamiento producido por la falta de comunicación [1].

Para ofrecer una solución auditiva a estos niños, se puede hacer uso de implantes cocleares o de audífonos. El implante coclear es un dispositivo electrónico capaz de transformar las señales acústicas 
recibidas en señales eléctricas que estimulan directamente el nervio auditivo, evitando las zonas afectadas. El implante no aumenta el sonido, sino que lo recoge y transforma para que el cerebro pueda procesarlo. Es por ello que la audición es diferente de la audición normal y se requiere cierto tiempo para aprender a oír [3]. En cambio, un audífono es un dispositivo electrónico que amplifica y modifica las señales sonoras [4].

El entrenamiento auditivo que realizan los educadores que trabajan con niños con problemas auditivos tiene cinco fases: detección, discriminación, identificación, reconocimiento y comprensión (ver Figura 1). Estas generalmente se solapan, llegando a ser trabajadas de manera simultánea en etapas más avanzadas. En la fase de detección, se comprueba si el niño detecta la presencia o ausencia de un sonido, variando la duración y la intensidad. Una vez el niño detecta el sonido, los educadores pueden trabajar habilidades auditivas como la discriminación o la identificación auditiva. La discriminación auditiva es la capacidad para identificar perceptivamente cualidades del sonido, como son unidades fonéticas y fonológicas relevantes en la comunicación o la duración o el timbre del sonido. El objetivo de la identificación es reconocer auditivamente un sonido o palabra dentro de un grupo (establecido previamente) y que el niño indique a cuál corresponde [5]. Finalmente, en la fase de reconocimiento, se comprueba si el niño discrimina sonidos o palabras de una lista abierta, tanto con apoyo visual como sin él, y, en la fase de comprensión, se trabajan aspectos como la capacidad de seguir una conversación o trabajar textos orales. En esta fase se comprueba si el niño puede reconocer un elemento verbal sin ayuda previa o participar en una conversación; es decir, se analiza si el niño puede procesar información que recibe auditivamente y decodificar el lenguaje [6]. En este trabajo nos centraremos especialmente en las fases segunda y tercera, que refieren a la identificación y discriminación.

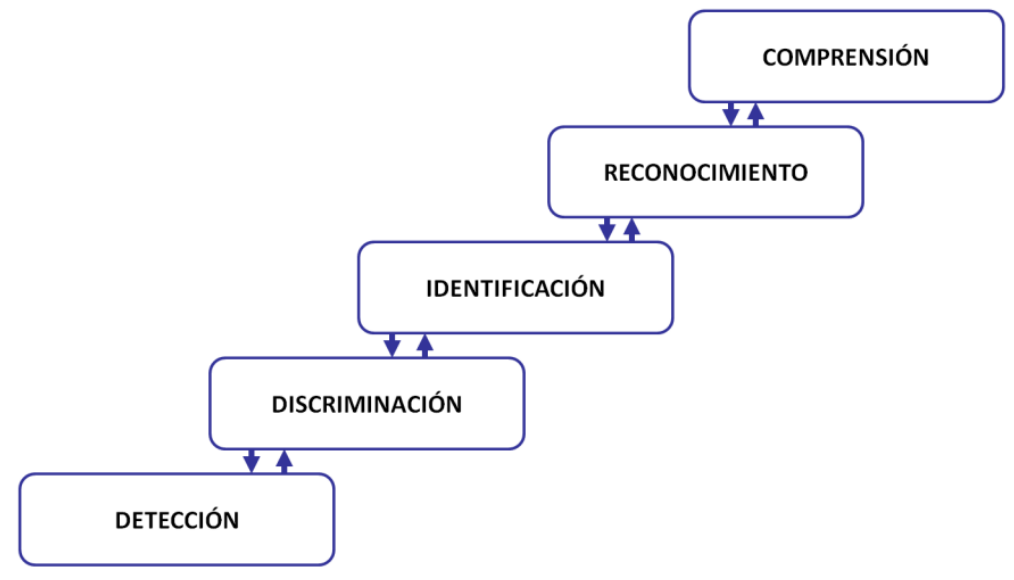

Figura 1. Fases del entrenamiento auditivo.

El entrenamiento de estas habilidades puede llegar a ser rutinario. Sin embargo, incluyendo aspectos afectivos y emocionales que son fundamentales en el comportamiento humano [7], se puede conseguir incrementar la motivación del niño, aumentar su interés por la tarea y, consecuentemente, que el niño aprenda estas habilidades de una forma más significativa. La unión de las emociones con el aprendizaje no es una idea nueva, Platón (428-348 a. C.) comentó que "todo aprendizaje tiene una base emocional". Por tanto, el proceso de enseñanza-aprendizaje no debe considerar únicamente la parte cognitiva, sino también la parte afectiva involucrada en este proceso.

En el campo del entretenimiento y del juego la parte emocional es fundamental. Las emociones están contempladas en el diseño de los juegos para poder crear experiencias positivas en los jugadores [8] y que éstos jueguen de forma voluntaria. Es por ello que está teniendo éxito la introducción de elementos de juego 
(gamification) en otros campos, como el de los negocios, deporte o educación, o el desarrollo de juegos con una finalidad más allá del entretenimiento (juegos serios). Además, en nuestro caso, las horas de clase o terapia tienen una duración limitada durante la semana. En cambio, si se ofrece una aplicación divertida, con la cual el niño puede jugar fuera de esas horas acordadas, el avance educativo puede mejorar.

Este trabajo presenta, por tanto, el diseño de una aplicación educativa cuyo objetivo es trabajar la discriminación e identificación auditiva en niños con discapacidad auditiva. En la segunda sección se presentan los trabajos relacionados con el diseño emocional y de juegos, metodologías de diseño de juegos serios y una recopilación de las actividades que realizan los educadores para trabajar la discriminación e identificación auditiva. La tercera sección describe el diseño del sistema, los elementos que lo componen y su funcionamiento. Finalmente, la última sección presenta las conclusiones y las líneas futuras de trabajo.

\section{Trabajos relacionadas}

Es un hecho que los sistemas diseñados al tener en cuenta aspectos afectivos y emocionales involucran a los estudiantes de forma positiva [9]. Hay que considerar diferentes aspectos a la hora de diseñar un juego serio en el campo de la educación y, más específicamente, en el campo de la educación para niños con discapacidad auditiva. Por un lado, tienen que tenerse en cuenta metodologías de diseño de juegos serios, la inclusión de elementos de juego y aspectos de diseño emocional. Por otro lado, hay que conocer el tipo de actividades que se incluyen dentro de las terapias cuyo objetivo es enseñar a los niños con problemas auditivos a discriminar e identificar sonidos y palabras. Posteriormente, hay que diseñar un espacio común donde la diversión, motivación o satisfacción personal del estudiante se equilibre con los objetivos buscados en las actividades de enseñanza/aprendizaje.

\subsection{Diseño emocional}

Este trabajo se basa en las definiciones de emoción como un "sentimiento general que se ve influenciado por estímulos internos y externos como el contexto, la experiencia pasada y actual o la personalidad". El afecto se define como "un sentimiento de corta duración, discreto, subjetivo y consciente" [10].

En [11] y [12], los autores presentan tres niveles de procesamiento en los humanos: visceral, conductual y reflexivo. Norman [6] asocia estos niveles a características de producto:

- Visceral (reacciones inducidas perceptualmente): apariencia.

- Conductual (reacciones inducidas por expectativas): placer y efectividad del uso.

- Reflexivo (reacciones inducidas intelectualmente): imagen de uno mismo, satisfacción personal y recuerdos.

Conocer quién será el usuario final del sistema a desarrollar puede ayudar a diseñarlo de forma que satisfaga los tres niveles. No obstante, debido a las diferencias individuales, culturales, o incluso físicas, el nivel de satisfacción no es el mismo en todos los usuarios ni tampoco predecible.

\subsection{Diseño de juegos serios en educación}

Los investigadores han analizado el diseño de juegos y, aunque la utilización de las recomendaciones encontradas no garantiza el diseño exitoso de un juego, sí es una buena práctica utilizarlas. Las recomendaciones a considerar incluyen listas de los ingredientes importantes que contienen videojuegos con éxito (auto-representación a través de avatares, rankings, niveles o competición bajo una reglas 
explícitas) [13], o utilización de mecanismos para implicar al jugador, como puntos, retos y misiones, o personalización [14].

Deterding et al. [15] presentaron una taxonomía de elementos de diseño de juego por nivel de abstracción, por ejemplo:

- Patrones de diseño de interfaz del juego: distintivos, tabla de clasificación, niveles.

- Heurísticas y principios de diseño: objetivos claros.

- Mecanismos y patrones de diseño de juegos: recursos limitados.

- Modelos de juego: retos.

- Métodos de diseño de juego: diseño centrado en el juego.

En el caso de los juegos serios para educación, además de tener en cuenta estos factores, también hay que incluir un componente pedagógico. Existe mucha investigación sobre metodologías para diseñar juegos serios para educación. Barbosa et al. [16] presentan una metodología compuesta por dos componentes: un juego principal con retos y un conjunto de mecanismos de aprendizaje que están relacionados con el juego, pero son independientes (p.ej. preguntas, puzles, mini juegos), por lo que es más fácil incluir contenidos de aprendizaje en el juego.

Marfisi-Schottman et al. [17] describen un método formado por siete pasos para diseñar juegos serios, marcando a los diferentes participantes del proceso las tareas a finalizar. Estas son: especificación de objetivos pedagógicos, selección del modelo de juego serio, descripción general del escenario, búsqueda de componentes de software, descripción detallada del escenario, control de calidad pedagógica y especificaciones para subcontratos.

Marne et al. [18] describen un marco de trabajo de seis fases, donde participan educadores y diseñadores de juegos. Estos autores diseñan un entorno cooperativo que permite a todos los participantes entender y comunicarse con un lenguaje común, a través de la creación de un lenguaje de patrones de diseño.

Prieto de Lope et al. [19] presentan una propuesta de metodología de desarrollo de juegos educativos basada en un guion interactivo, dividido en capítulos y escenas, que integra todos los aspectos trasversales del juego.

En la línea del público objetivo del juego serio que se presenta en este trabajo, Cano et al. [20] describen una metodología específica para el diseño de juegos serios para niños con discapacidad auditiva basada en 4 fases: análisis, pre-producción, producción y post-producción. Esta metodología ha sido utilizada para el diseño de productos enfocados a niños con discapacidad auditiva, con diferentes objetivos educativos [21]-[23]. También, el trabajo de Peñeñory et al. [24] presenta una propuesta de un modelo para el análisis de requisitos y actividades para el diseño de experiencias interactivas dirigidas a la rehabilitación psicomotriz de niños con discapacidad auditiva. Este modelo fusiona las técnicas y herramientas más importantes provenientes del diseño centrado en el usuario (DCU) y la teoría de la actividad, que permite realizar un análisis más profundo de todas las variables, para poder establecer cuáles son las funcionalidades de la experiencia interactiva que mejor se adaptan a las actividades y las motivaciones de las personas con discapacidad auditiva en rehabilitación psicomotriz.

\subsection{Juegos serios para educación de niños con problemas auditivos}

Yi y Kim [25] presentan un juego para niños con problemas auditivos que, debido a su disfunción comunicativa, tienen dificultades para aprender las reglas sociales. En el juego, introducen elementos de iniciativa (creando una historia interesante, variando los personajes), de continuidad (para que los niños jueguen más tiempo) y de cumplimiento de objetivos (competición y recompensas) para el entrenamiento auditivo.

Cano et al. [22] presentan un juego que recrea una historia que hace progresar al niño por diferentes niveles, que le ayudan a desarrollar habilidades para escuchar y hablar. 
Alamoudi et al. [26] presentan el diseño conceptual de un juego para discriminación auditiva, en lengua árabe y con diferentes dialectos.

\subsection{Actividades para identificación y discriminación}

Para trabajar las habilidades de discriminación, se pueden realizar diferentes actividades [27]:

- Jugar a presentar al niño dos sonidos. Este tiene que indicar el que ha oído, bien señalando una imagen u objeto, bien repitiéndolo (especialmente si desconoce la palabra). Estos sonidos pueden provenir, por ejemplo, de una campana o un tambor, o pueden ser sonidos como 'oh' o 'ah'. También es necesario comprobar que el niño oye sonidos de consonantes, al inicio o al final de las palabras, y de frecuencias diferentes, por ejemplo: de alta frecuencia como la /s/, o de baja frecuencia como la $/ \mathrm{m} /$ [28].

- Jugar con el volumen o longitud. Si el niño está imitando lo que oye, se podrá observar si detecta la diferencia.

- Jugar a localizar el sonido, identificar de dónde proviene.

Para trabajar las habilidades de identificación, se pueden realizar diferentes actividades:

- Jugar a presentar al niño dos palabras iguales o parecidas fonéticamente como 'blanco' y 'banco', para analizar si el niño identifica las diferentes palabras. Inicialmente, también se le pueden presentar dos palabras que difieran en número de sílabas.

\section{Diseño del sistema}

El diseño del sistema se ha realizado siguiendo la filosofía de diseño centrado en el usuario (ver Figura 2). Este enfoque de diseño y desarrollo de sistemas interactivos tiene como objetivos obtener sistemas más usables y aplicar técnicas y conocimientos de ergonomía y usabilidad.

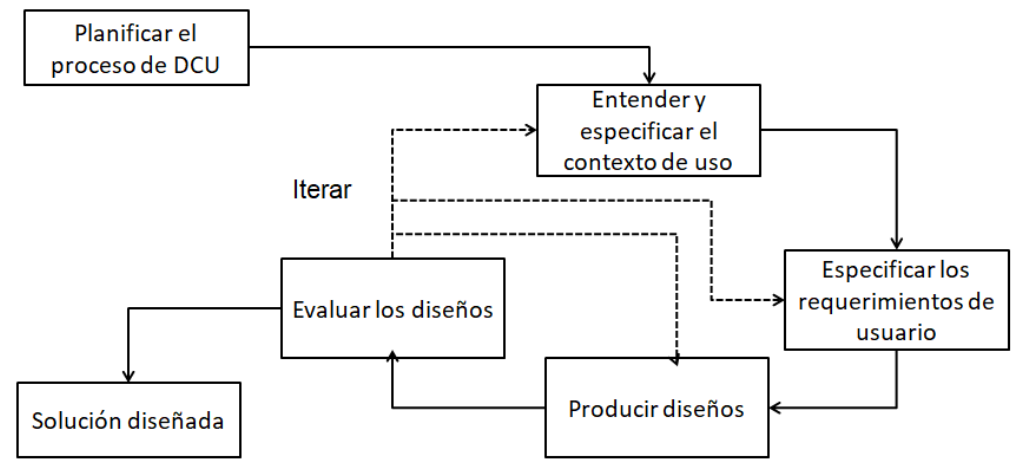

Figura 2. Actividades del DCU [29].

En el diseño del sistema y de las actividades educativas participó un grupo multidisciplinar: profesionales de escuelas y centros que atienden población con problemas auditivos, diseñadores, ingenieros informáticos y electrónicos.

Se llevó a cabo observación de sesiones en los centros con niños con problemas auditivos, se entrevistó a los educadores para recoger requisitos sobre las actividades educativas y se han recogido las impresiones de los usuarios finales respecto al diseño gráfico y el primer prototipo del sistema. 
El sistema se compone (1) de un tablero físico de juego, con unas figuras que representan a cada uno de los jugadores que participan y (2) una app para dispositivos móviles Android (tablets), que simula el tablero y ejecuta las actividades educativas. El juego está preparado para poder ser jugado tan solo con la app, si no se cuenta con el tablero físico.

En el diseño, se busca englobar las características del producto mencionadas por Norman: apariencia, placer, efectividad de uso, imagen de uno mismo, satisfacción personal y recuerdos. También integra aspectos educativos lúdicos, creativos e interactivos. La dimensión educativa está cubierta por las actividades recopiladas por los educadores para trabajar la identificación y discriminación auditiva: son los retos presentados dentro del juego. El entretenimiento y la creatividad se consiguen a través de la creación de una historia y unos personajes, que consiguen la inmersión del niño en el juego, le permiten trabajar su imaginación y lo introducen dentro de una aventura con objetivos claros. Además, cuenta con dos niños con implante coclear como personajes principales, de diferente género, llamados Gabriel y Gabriela y conocidos como Gaby, de modo que el niño o niña pueda identificarse con alguno de ellos. Por esto, tal cantidad de elementos, junto con la manera cómo interactúa el juego al combinar elementos tangibles con digitales, motivan al niño a tocar elementos físicos y a aumentar su experiencia con información digital, presentada en una tablet.

\subsection{Historia}

La historia presenta un conjunto de personajes y mundos que buscan que el niño se adentre en la aventura. La historia se creó en Colombia y, aunque tiene una estructura y un contenido bastante universal, han sido necesarias pequeñas adaptaciones culturales al contexto español. El protagonista de la historia es un niño o niña, cuyo nombre inicialmente es Gaby, para poder adaptarse a un personaje tanto masculino como femenino. Ahora bien, en las evaluaciones previas de los educadores en España, específicamente en la comunidad de las Islas Baleares, uno de los primeros requisitos fue poder adaptar los nombres de los personajes a nombres más familiares para los niños con los que se trabajaba. Incluso, hay diferencias entre centros, ya que los centros de las zonas interiores preferirían las versiones mallorquinas de los nombres y, en la ciudad, donde hay más castellano-parlantes, las versiones españolas.

A su vez, existen personajes colaboradores o mentores, que ayudan al personaje principal, y un personaje malvado, al cual hay que derrotar.

\subsection{Tablero físico}

El diseño gráfico del tablero fue un proyecto de diseño, desarrollado [23] a través de brainstorming y design thinking [30], para incluir creatividad y diversión (historia, personajes), estética (lenguaje visual y auditivo, colores vivos, elementos reconocibles por los niños, como montañas, bosques, volcanes, animales) y empatía (familiaridad con los personajes) (ver Figura 3).

El tablero físico presenta un camino, que representa el oído interno, de seis a ocho baldosas del mismo color, que indican ocho niveles de dificultad creciente. Para cada nivel de dificultad existe una tarjeta con una etiqueta RFID, que será leída por el lector RFID, que a su vez se comunicará por medio de bluetooth a la tablet, para ejecutar una actividad educativa diferente dentro de ese nivel, mientras el jugador no lo supere. Por ello, también existe una "caja mágica", un lector de RFID, a la cual el jugador tiene que acercar la tarjeta RFID correspondiente, cuando sea su turno. Cuenta con figuras modeladas en forma de niños con implantes cocleares que personifican a cada uno de los jugadores que participan.

El diseño gráfico del tablero y de los personajes principales fue evaluado, en un proyecto anterior, en el Instituto de Niños Ciegos y Sordos del Valle del Cauca, Colombia y adaptado a los requisitos de nuestro caso [23], [31]. 


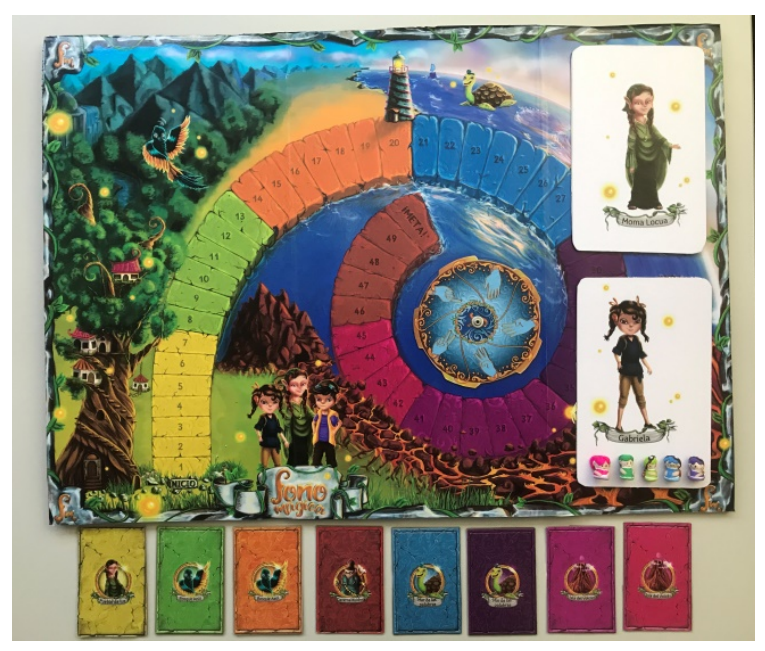

Figura 3. Tablero físico, con tarjetas RFID, figuras de jugadores y tarjetas de personajes.

\subsection{App educativa}

La app está desarrollada para dispositivos Android e incluye adaptación automática a las diferentes resoluciones de pantalla de los múltiples dispositivos disponibles en el mercado. Se ha utilizado Android Studio para su desarrollo y depuración.

La app es completamente autocontenida, por lo que permite jugar sin necesidad del tablero físico, pues este queda representado en la app (ver Figura 4). Inicialmente, se selecciona el número de jugadores y cada jugador puede dar un nombre a su personaje (ver Figura 5). Todos los jugadores seleccionados se pondrán en la línea de salida. Hay que destacar que el juego puede ser multijugador, o puede ser utilizado con un único jugador con el apoyo del educador.

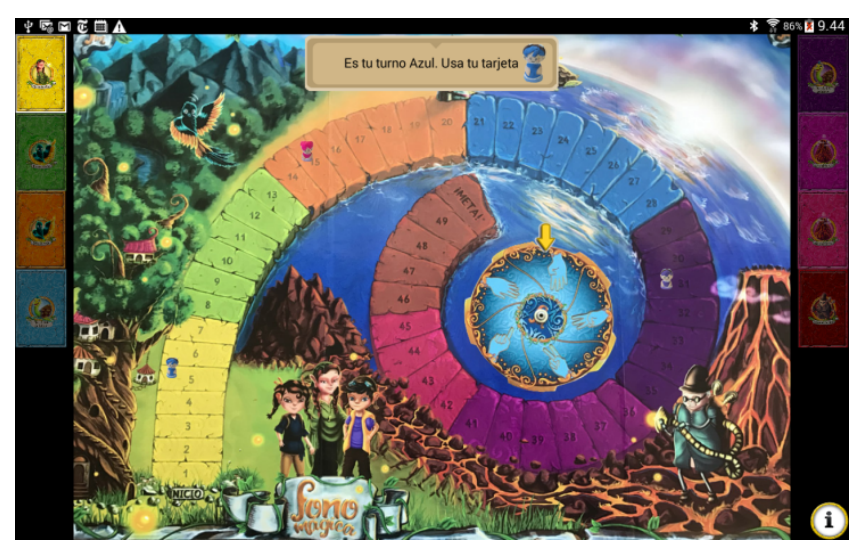

Figura 4. App para trabajar la discriminación auditiva.

En el turno de cada jugador, se le presentarán tareas educativas de identificación de sonidos y discriminación de palabras. Si acierta un número predefinido de palabras, entonces hará rodar una ruleta para conocer cuántas casillas deberá moverse. En caso contrario, se quedará en su posición actual. 


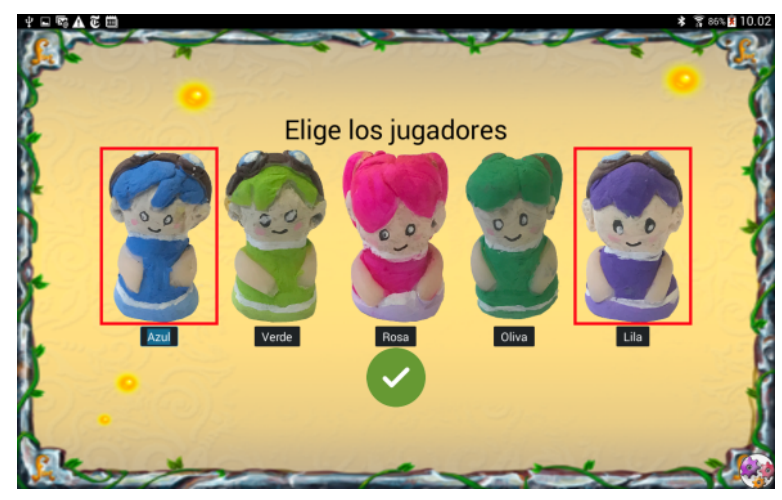

Figura 5. Selección de número de jugadores y configuración del nombre.

Los sonidos a discriminar son, por ejemplo, /b/-/m/, /b/-/f/. Están incluidos en parejas de palabras que lo niños tiene que identificar. A continuación, se listan algunas parejas de palabras que se trabajan:

- $\quad / \mathrm{b} /-/ \mathrm{m} /$ : bota-mota, bote-mote, besa-mesa, bar-mar, daba-dama, calva-calma, desván-desmán.

- /b/-/f/: base-fase, boca-foca, veo-feo, balda-falda, baba-faba, vino-fino.

- /ch/-/p/: chapa-papa, choza-poza, chillar-pillar, chino-pino, choca-poca, chata-pata.

La app verbaliza de forma aleatoria una de las palabras de cada pareja (organizadas por niveles de dificultad) y, en la pantalla, aparecen las dos palabras a discriminar (ver Figura 6). El niño deberá seleccionar aquella que haya identificado. Si la palabra es acertada, se le indicará con una retroalimentación positiva. Las palabras a identificar y discriminar han sido proporcionadas por los educadores de un centro de atención a personas con discapacidad auditiva en España, a partir de ejercicios que se realizan en el centro. En ese caso, el educador pronuncia las palabras y el niño repite la palabra que ha oído.
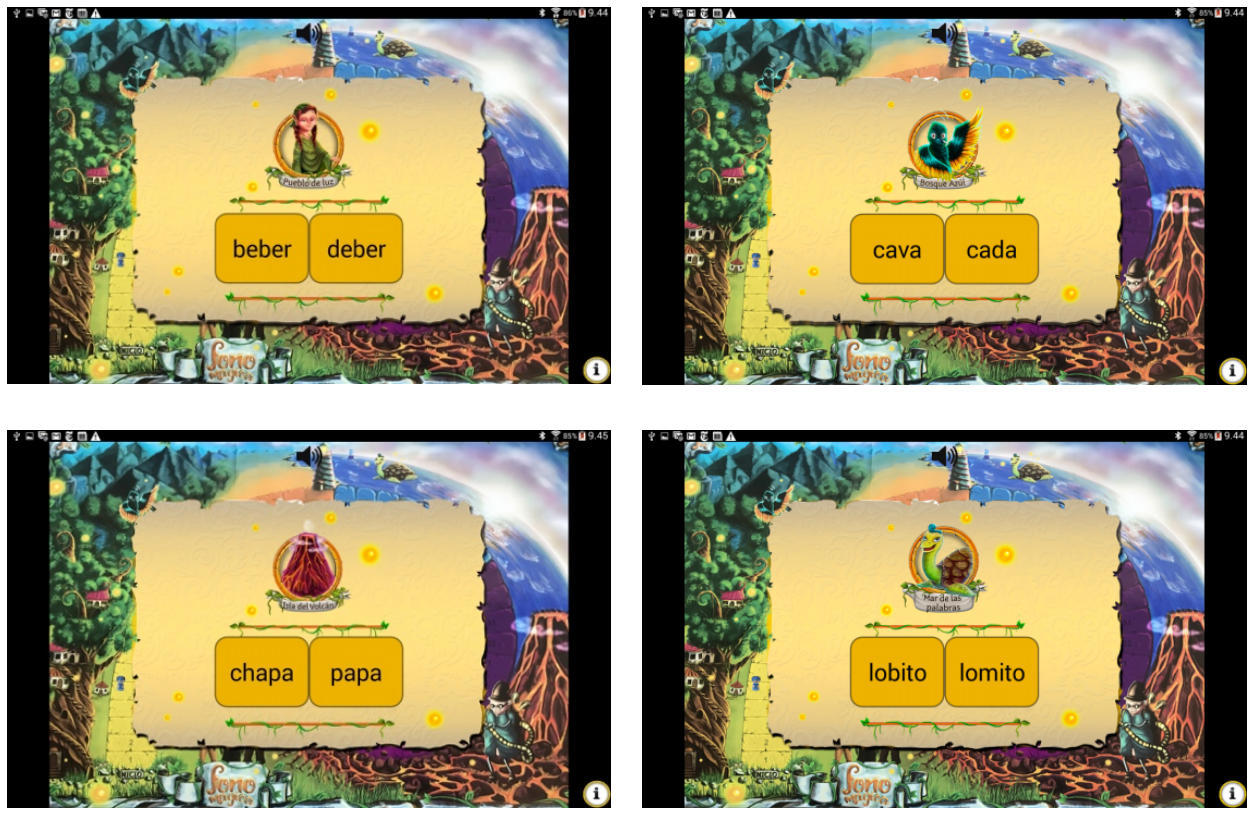

Figura 6. Tareas de identificación y discriminación de palabras. Fila de arriba: dos ejercicios diferentes para trabajar la /b/-/d/. Fila de abajo: izquierda /ch/-/p/, derecha: /b/-/m/. 
Para ofrecer un sistema flexible y adaptable a cada niño, el educador puede configurar los sonidos a identificar en cada uno de los niveles, según el niño con el que se juegue (ver Figura 7). Puede poner las identificaciones/discriminaciones más sencillas en los primeros niveles y aumentar la dificultad, a medida que evolucione el juego.

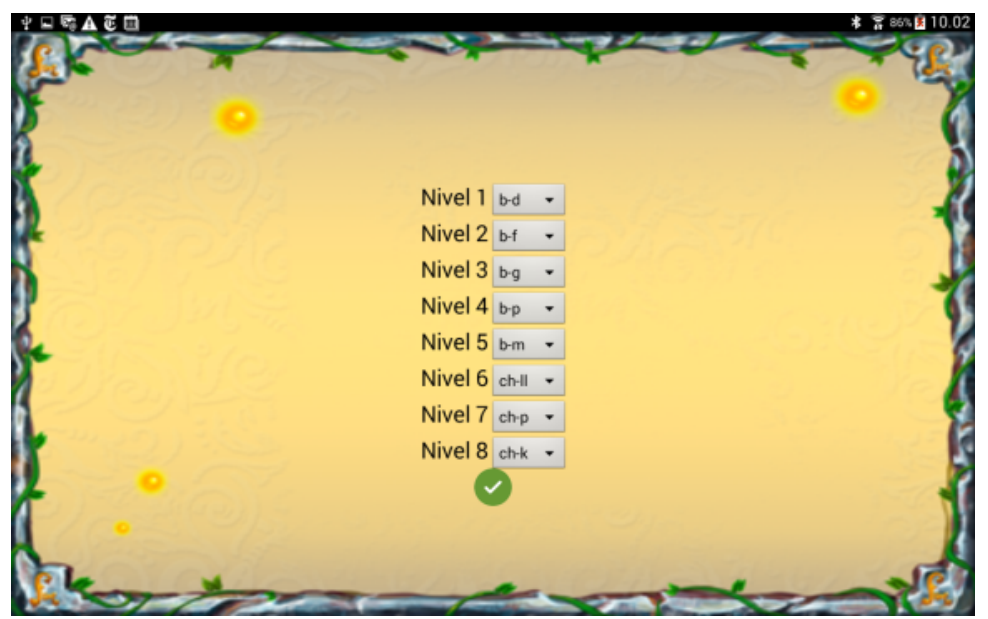

Figura 7. Configuración de cada nivel.

Además, la aplicación guarda datos sobre las sesiones de trabajo, para poder analizarlos más adelante, así como para incluir nuevas combinaciones de fonemas o más palabras y ver el progreso del niño.

Tecnológicamente, la aplicación se apoya en una estructura de datos organizada por categorías de discriminación, en cada una de las cuales se almacenan las palabras determinadas por el equipo de educadores. La flexibilidad de esta estructura permite su fácil ampliación, almacenaje en una base de datos SQLite y actualización remota, haciendo posible añadir fácilmente, en una futura versión, categorías y vocablos del entorno, sin necesidad de recompilar la aplicación.

En la versión actual, las palabras se vocalizan utilizando la clase TextToSpeech, lo que confiere a la aplicación una gran flexibilidad pero que, al mismo tiempo, introduce un halo de artificialidad que los educadores han considerado mejorable. Por este motivo, se está procediendo a la grabación, utilizando diferentes voces, de las palabras almacenadas.

Para facilitar la visualización del tablero y la adaptación de los avatares a las posiciones de cada una de las casillas, se utilizan regiones de delimitación que garantizan una transición sencilla a nuevos niveles de juego, que puedan introducir tableros de diferente diseño y organización. Las regiones se han definido a partir de desplazamientos relativos entre ellas, por lo cual una reorganización de las casillas, simplemente, implica un ligero ajuste interactivo en las transformaciones geométricas entre ellas, para permitir su nuevo cálculo (ver Figura 8).

Una parte importante del tablero es la ruleta, con la cual el usuario determina el número de casillas que debe avanzar su avatar. Se ha implementado mediante una imagen superpuesta a la ruleta del tablero, con la que se simula, cinemáticamente, el movimiento resultante del impulso inicial que se imprime al arrastrar el dedo sobre ella. Las posiciones inicial y final del arrastre, junto con el tiempo invertido en realizar todo el movimiento, sirven para determinar la aceleración inicial que determinará el comportamiento resultante en la simulación. La puntuación obtenida con un lanzamiento se determina por la posición final resultante del movimiento (ver Figura 9). 


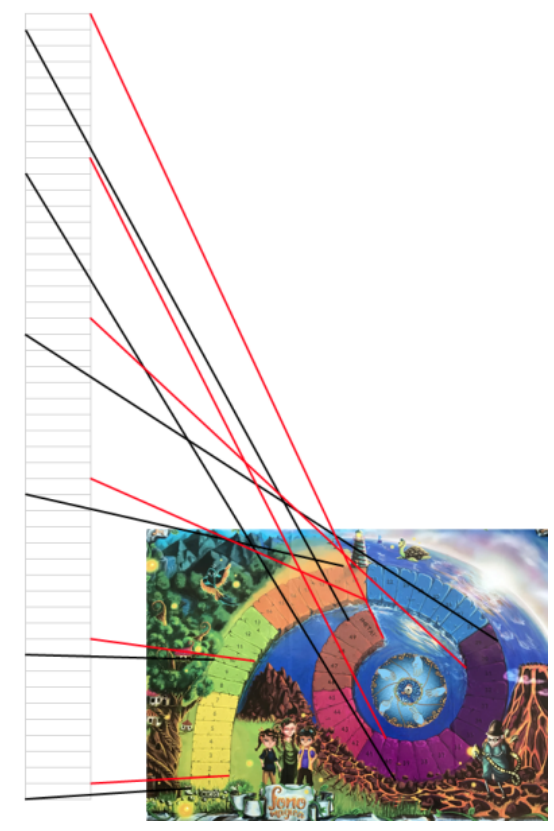

Figura 8. Correspondencias geométricas para las casillas de juego (regiones de delimitación) para la determinación automática de la posición de los avatares.
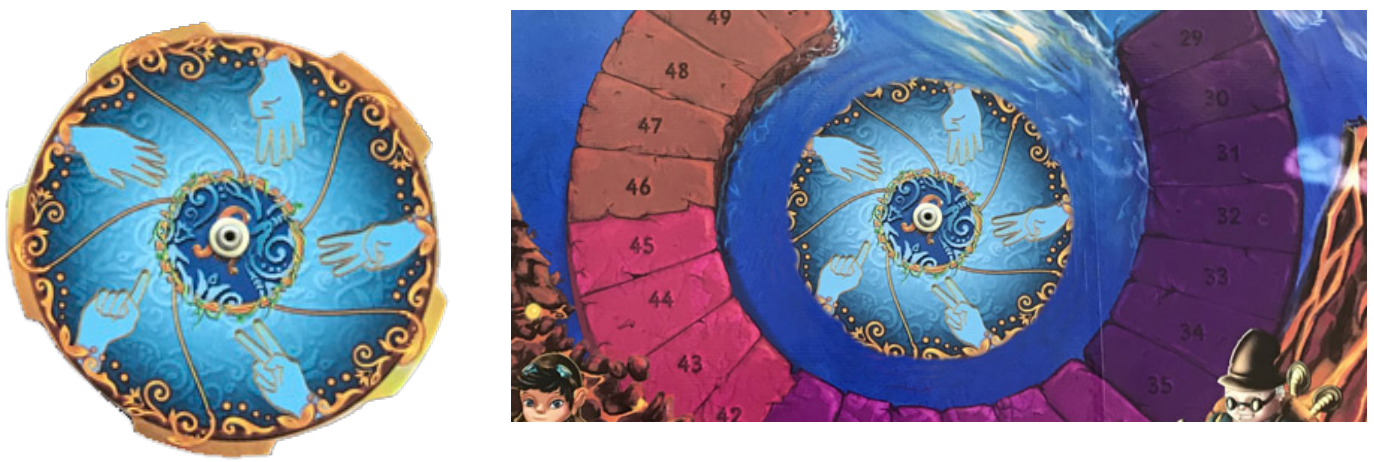

Figura 9. La simulación del movimiento de la ruleta se consigue con una superposición de una imagen sobre el tablero original.

\subsection{Evaluación inicial}

El tablero y la app se mostraron a los educadores de ASPAS Mallorca. Además de realizar las modificaciones en los nombres de los personajes para la historia, se comentó que sería importante que la vocalización de las palabras se realizase con voz humana. Específicamente, se solicitó grabar las palabras con voz de mujer, voz de hombre y voz susurrada.

Después, se realizó una primera prueba con usuarios reales: cuatro niños ( 2 niños y 2 niñas), cuyas edades están entre 8 y 9 años. Los niños comentaban aspectos que les gustaban, como poder identificar a su jugador con un nombre propio, o poder ver el tablero físico representado en la app. Después de jugar con el sistema, se les pasó un cuestionario para rellenar. Tres de los niños asociaron la emoción de alegría a su experiencia con el juego y uno de ellos la emoción neutral. Esto corresponde con su contestación a la pregunta “¿disfrutaste de la experiencia?, ¿ha sido divertida?”, a la que tres respondieron con "mucho” y uno "poco". Ninguno marcó la emoción de disgusto. Los niños jugaron de forma grupal y se observó que se ayudaban entre sí para conseguir los objetivos (ver Figura 10). 


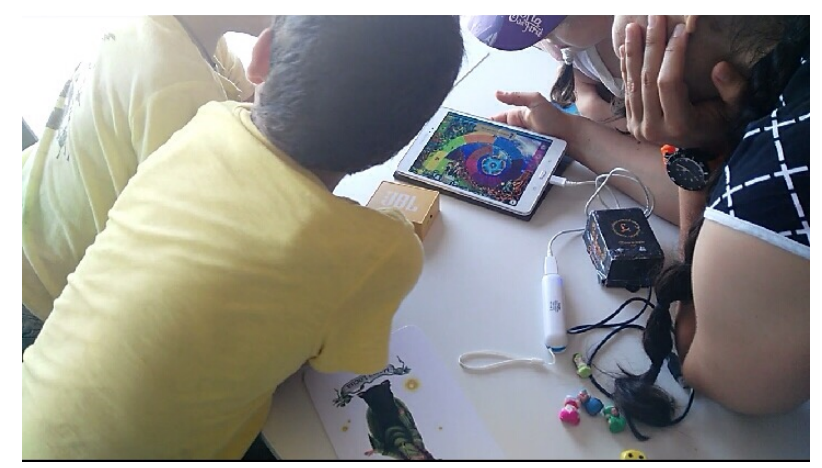

Figura 10. Niños evaluando el sistema. ASPAS - Mallorca.

\section{Conclusiones y trabajo futuro}

En este trabajo se ha presentado el diseño de un juego serio, cuyo objetivo es trabajar la identificación y la discriminación auditiva en niños con discapacidades auditivas. El diseño ha sido realizado por un grupo multidisciplinar, que engloba profesionales del diseño, electrónica, informática, educadores de niños con problemas auditivos. También se han introducido a los usuarios finales en las fases de evaluación. En el diseño, se ha buscado el equilibrio entre las actividades pedagógicas y las lúdicas. Se ha creado una historia con personajes con los que los niños puedan empatizar, así como con personajes colaboradores y antagónicos. Se presenta una estética del agrado de los niños con los que se trabaja, buscando responder a las características consideradas en el diseño emocional.

El sistema se compone de un tablero y fichas físicas, combinado con una app instalada en una tablet, donde se presentarán las actividades pedagógicas, retos y animaciones. Este diseño permite mucha flexibilidad en la configuración de los niveles o de los nombres de personajes, o en la inclusión de nuevo vocabulario o fonemas a trabajar. Además, el sistema está preparado para jugar únicamente con la app en la tablet si no se dispone del tablero físico.

Como trabajo futuro, es importante realizar las grabaciones de las palabras con voces humanas, de mujer, de hombre y voz susurrada. También es necesario realizar una evaluación más profunda, junto a los niños tanto de los centros españoles, como de los colombianos. Inicialmente, las actividades pedagógicas introducidas son ejercicios trabajados actualmente en el centro español y fueron facilitadas por los educadores. Por ello, es posible que las palabras deban adaptarse al contexto colombiano y deban cambiarse. Además, se está colaborando con un grupo español de pedagogía aplicada y psicología de la educación, que tiene un proyecto con un centro para niños sordos en Marruecos, interesados en probar el sistema. Probablemente, en este nuevo contexto cultural, se tendrán que hacer modificaciones tanto en la parte de contenido (nombres de los personajes de la historia, palabras), como en la parte estética.

\section{Agradecimientos}

Este trabajo ha sido parcialmente financiado por TIN2016-81143-R (AEI/FEDER, UE) y el proyecto OCDS-CUD2016/13, financiado por la Universitat de les Illes Balears. También se agradece a P. Giraldo Bustamante y L. Galves Cubillos por el trabajo de diseño gráfico y a los educadores y niños de ASPAS Mallorca (España) y del Instituto de niños ciegos y sordos del Valle del Cauca (Colombia) por su ayuda. 


\section{Referencias}

[1] World Health Organization, "Fact sheet. Deafness and hearing loss. Disponible en: http://www.who.int/ mediacentre/factsheets/fs300/en/." 2017.

[2] V. M. Peñeñory, C. Manresa-Yee, I. Riquelme, C. A. Collazos, H. M. Fardoun, and D. M. Alghazzawi, "Review of systems to train psychomotor skills in hearing impaired children," in Proc. 4th Workshop on ICTS for improving patients rehabilitation research techniques, 2016, pp. 81-84.

[3] National Institute on Deafness and other Communication disorders, "Implantes cocleares". Disponible en: https:// www.nidcd.nih.gov/es/espanol/implantes-cocleares. [Accedido: Feb. 2017].

[4] National Institute on Deafness and other Communication disorders, "Audífonos". Disponible en: https:// www.nidcd.nih.gov/es/espanol/audifonos. [Accedido: Feb. 2017].

[5] G. González, "Logopedia Escolar Digitalizada. Informe Series Desarrollo, transferencia y difusión social de la investigación en TIC para la Educación". Ministerio de Educación, Políticasocial y deporte. Instituto Superior de Formación y Recursos en Red para el Profesora.

[6] ASPAS Mallorca, "Fases del entrenamiento auditivo". Disponible en: http://www.aspasleehablacomunica.com/ fases-del-entrenamiento-auditivo/. [Accedido: 01/12/2017].

[7] D. Norman, Emotional Design: Why We Love (or Hate) Everyday Things. 2004.

[8] G. N. Yannakakis, K. Karpouzis, A. Paiva, and E. Hudlicka, "Emotion in Games," in Affective Computing and Intelligent Interaction: Fourth International Conference, ACII 2011, Memphis, TN, USA, October 9--12, 2011, Proceedings, Part II, S. D’Mello, A. Graesser, B. Schuller, and J.-C. Martin, Eds. Berlin, Heidelberg: Springer Berlin Heidelberg, 2011, p. 497.

[9] P. A. Schutz and R. Pekrun, Chapter 1 - Introduction to Emotion in Education. Burlington: Academic Press, 2007.

[10] T. Bentley, L. Johnston, and K. von Baggo, "Evaluation Using Cued-recall Debrief to Elicit Information About a User's Affective Experiences," in Proceedings of the 17th Australia Conference on Computer-Human Interaction: Citizens Online: Considerations for Today and the Future, 2005, pp. 1-10.

[11] D. A. Norman, A. Ortony, and D. M. Russell, "Affect and machine design: Lessons for the development of autonomous machines," IBM Syst. J., vol. 42, no. 1, pp. 38-44, 2003.

[12] D. Norman and A. Ortony, "Designers and Users: Two Perspectives on Emotion and Design," in Proc. of the Symposium on "Foundations of Interaction Design" at the Interaction Design Institut, 2003.

[13] B. Reeves and J. . Read, Total Engagement: Using Games and Virtual Worlds to Change the Way People Work and Businesses Compete. Harvard Business School Press, 2009.

[14] G. Zichermann and C. Cunningham, Gamification by Design. O'Reilly Media, Inc., 2011.

[15] S. Deterding, D. Dixon, R. Khaled, and L. Nacke, "From Game Design Elements to Gamefulness: Defining "Gamification,"' in Proceedings of the 15th International Academic MindTrek Conference: Envisioning Future Media Environments, 2011, pp. 9-15.

[16] A. F. S. Barbosa, P. N. M. Pereira, J. A. F. F. Dias, and F. G. M. Silva, "A New Methodology of Design and Development of Serious Games,” Int. J. Comput. Games Technol., vol. 2014, no. Article ID 817167, p. 8 pages, 2014.

[17] I. Marfisi-Schottman, S. George, and F. Tarpin-Bernard, "Tools and Methods for Efficiently Designing Serious Games"," in 4th Europeen Conference on Games Based Learning ECGBL2010, Copenhagen, Denmark, 21-22 October 2010, 2010, pp. 226-234.

[18] B. Marne, J. Wisdom, B. Huynh-Kim-Bang, and J.-M. Labat, "The Six Facets of Serious Game Design: A Methodology Enhanced by Our Design Pattern Library," in 21st Century Learning for 21st Century Skills: 7th European Conference of Technology Enhanced Learning, EC-TEL 2012, Saarbr\{ü\}cken, Germany, September 18-21, 2012. Proceedings, A. Ravenscroft, S. Lindstaedt, C. D. Kloos, and D. Hernández-Leo, Eds. Berlin, Heidelberg: Springer Berlin Heidelberg, 2012, pp. 208-221.

[19] R. Prieto de Lope, N. Medina-Medina, P. Paderewski, and F. L. Gutiérrez Vela, "Design Methodology for Educational Games based on Interactive Screenplays.," in CoSECivi, 2015, vol. 1394, pp. 90-101.

[20] S. Cano, J. M. Arteaga, C. A. Collazos, C. S. Gonzalez, and S. Zapata, "Toward a methodology for serious games design for children with auditory impairments," IEEE Lat. Am. Trans., vol. 14, no. 5, pp. 2511-2521, May 2016.

[21] S. Cano, C. A. Collazos, C. Manresa-Yee, and V. Peñeñory, "Principles of Design for Serious Games to Teaching of Literacy for Children with Hearing Disabilities," in Proceedings of the XVII International Conference on Human Computer Interaction, 2016, p. 6:1-6:2.

[22] S. Cano, V. Peñeñory, C. A. Collazos, H. M. Fardoun, and D. M. Alghazzawi, "Training with Phonak: Serious Game As Support in Auditory -- Verbal Therapy for Children with Cochlear Implants," in Proceedings of the 3rd 2015 Workshop on ICTs for Improving Patients Rehabilitation Research Techniques, 2015, pp. 22-25. 
[23] S. Cano, L. Galvez, P. Giraldo, C. Collazos, and H. Fardaun, "Sistema interactivo para la enseñanza de la lectoescritura para niños con implante coclear," Rev. Iberoam. Inform. Educ., no. 4, pp. 21-29, 2016.

[24] V. M. Peñeñory, C. Manresa-Yee, C. A. Collazos, S. Cano, and H. M. Fardoun, "Propuesta de modelo para el análisis de requisitos y actividades para el diseño de experiencias interactivas dirigidas a la rehabilitación psicomotriz de niños con discapacidad auditiva," in 18th International Conference on Human Computer Interaction, Cancun, Quintana roo MEXICO, September 2017 (Interaccion'17)(México, 25-27 September 2017), 2017.

[25] C. Yi and T. Kim, "Serious Game Design for Auditory Training of HearingImpaired Children," TechArt J. Arts Imaging Sci., vol. 2, no. 1, pp. 46-51, 2015.

[26] A. Alamoudi, M. Almozaini, R. Alabdulrahman, S. Alkoblan, S. Drine, and A. Al-Wabil, "Interactive Serious Gaming for Children with Auditory Processing Difficulties in the Arabic Language," in HCI International 2013 Posters' Extended Abstracts: International Conference, HCI International 2013, Las Vegas, NV, USA, July 21-26, 2013, Proceedings, Part I, C. Stephanidis, Ed. Berlin, Heidelberg: Springer Berlin Heidelberg, 2013, pp. 188-191.

[27] N. Fullana Rivera, "Age-related effects on the acquisition of a foreign language phonology in a formal setting. Appendix A,” Universitat de Barcelona, 2006.

[28] K. B. Agung, S. C. Purdy, and C. Kitamura, "The Ling Sound Test Revisited," Aust. New Zeal. J. Audiol., vol. 27, no. 1, pp. 33-41, 2005.

[29] ISO 9241-210:2010, "Ergonomics of human-system interaction - Part 210: human-centred design for interactive systems," Int. Organ. Stand., 2010.

[30] K. Dorst, “The core of 'design thinking' and its application,” Des. Stud., vol. 32, no. 6, pp. 521-532, Nov. 2011.

[31] "Fonomágica". Disponible en: http://www.fonomagica.co. [Accedido: Marzo 2017].

\section{Sobre los autores}

\section{Cristina Manresa-Yee}

Doctora en Informática por la Universitat de les Illes Balears (2009). Actualmente es profesora de la Universitat de les Illes Balears e investigadora dentro del grupo Unidad de gráficos, visión por computador e inteligencia artificial. Sus líneas de investigación son la interacción persona-ordenador, visión por computador y ayudas técnicas para usuarios con necesidades especiales. Es miembro de la Asociación Interacción Persona-Ordenador (AIPO).

\section{Ramón Mas Sansó}

Licenciado en informática por la Universidad Autónoma de Barcelona y doctor en Informática por la Universitat de les Illes Balears (1996). Actualmente trabaja como profesor titular en la Universitat de les Illes Balears y forma parte del equipo investigador del grupo Unidad de gráficos, visión por computador e inteligencia artificial. Sus líneas de investigación son la interacción persona-ordenador, los gráficos por ordenador y ayudas técnicas para usuarios con necesidades especiales.

\section{Sandra Cano}

Ingeniera electrónica en la Universidad Autónoma de Occidente de Cali, y doctora en Ciencias de la Electrónica en la Universidad del Cauca, Colombia, forma parte del equipo investigador LIDIS de la Universidad San Buenaventura de Cali. Sus líneas de investigación son la interacción persona-ordenador, computación cognitiva e inclusión digital y social para usuarios con necesidades especiales. Es miembro de la Asociación Interacción Persona-Ordenador (AIPO). 